\title{
Apprendre une langue quand on en parle déjà une
}

Quels enseignements pour quels apprentissages à l'école primaire?

\section{Michèle Garabédian}

\section{OpenEdition}

\section{Journals}

Édition électronique

URL : http://journals.openedition.org/ries/3404

DOI : $10.4000 /$ ries.3404

ISSN : 2261-4265

Éditeur

Centre international d'études pédagogiques

Édition imprimée

Date de publication : 1 mars 1996

Pagination : 119-130

ISSN : $1254-4590$

Référence électronique

Michèle Garabédian, «Apprendre une langue quand on en parle déjà une », Revue internationale d'éducation de Sèvres [En ligne], 09 | 1996, mis en ligne le 20 août 2013, consulté le 23 mars 2021. URL : http://journals.openedition.org/ries/3404; DOI : https://doi.org/10.4000/ries.3404

Ce document a été généré automatiquement le 23 mars 2021

(c) Tous droits réservés 


\title{
Apprendre une langue quand on en parle déjà une
}

Quels enseignements pour quels apprentissages à l'école primaire?

\author{
Michèle Garabédian
}

\section{Un constat}

1 L'enseignement des langues étrangères dans le premier degré de la scolarité est aujourd'hui un secteur en plein développement. De nombreux signes en portent témoignage, à commencer par le domaine de l'édition, qui n'est pas souvent, dans le domaine des langues, le plus audacieux. En effet, depuis quelques années, une vitrine internationale comme Expolangues a fait une place de plus en plus importante aux matériels didactiques destinés à ce jeune public.

2 Présent dès 1990 dans le programme officiel d'Expolangues, ce domaine des langues semble gagner certaines lettres de noblesse puisqu'en 1996, il fait partie des priorités retenues dans le cadre d'Innovalangues, concours sélectionnant et récompensant les projets d'innovation par les organisateurs du $14^{\mathrm{e}}$ Expolangues ${ }^{1}$.

3 Les catalogues des éditeurs de langues ne sont pas les seuls à témoigner de la vigueur de ce secteur "porteur "; les nombreux colloques, rencontres pédagogiques, sessions de formation qui fleurissent sans désemparer depuis cinq ans sur cette thématique en sont aussi des marques objectives.

4 On peut constater qu'au-delà des effets d'annonce et de nouveauté qu'avait engendrés un certain nombre de décisions politiques concernant la mise en place de l'enseignement d'une langue étrangère dès l'école primaire, le mouvement perdure, s'élargit et des perspectives de généralisation sont envisagées, à moyen terme, dans un grand nombre de pays européens tels que l'Italie, la France, l'Espagne, la Grèce.

5 Si l'intérêt n'a pas faibli, au contraire, si les expériences se sont multipliées, cela ne veut pas dire pour autant que les choses soient acquises. En effet, si le principe d'une introduction des langues étrangères à l'école primaire semble susciter une attitude 
favorable des parents et recueillir un certain consensus auprès de l'opinion publique, il n'en reste pas moins un grand nombre de difficultés à surmonter et de questions à résoudre, et pas des moindres, pour que cette introduction devienne une innovation.

La généralisation d'un phénomène, voire sa mondialisation, n'en fait pas automatiquement une innovation pédagogique, voire une avancée éducative. Il est donc important de s'interroger sur les enjeux d'une telle décision.

\section{Des questions en forme de propositions}

\section{À propos des politiques linguistiques}

7 Si l'on décide que l'école primaire entre dans le dispositif que se donne un État, à travers son système éducatif, pour développer la place des langues dans la société, il faut que les responsables politiques en assument toutes les conséquences.

Introduire l'enseignement d'une langue étrangère dès l'école primaire ne constitue-t-il pas, à long terme, un facteur de progrès démocratique sachant qu'il va toucher la plus forte cohorte d'enfants, à un stade où l'éducation est un phénomène de masse ?

Introduire l'enseignement d'un idiome inconnu dans des centaines de milliers d'écoles primaires ne représente-t-il pas une chance, pour toute société, de regarder en face ses atouts mais aussi ses ignorances dans le domaine des langues?

L'école primaire, rouage essentiel dans le fonctionnement d'une société, pourra-t-elle rajouter à son cahier des charges, un quatrième apprentissage fondamental - après lire, écrire et compter, apprendre une langue étrangère - sans qu'un débat public s'instaure sur la place des langues dans ladite société, dans les échanges transnationaux, dans l'ensemble du cursus scolaire, dans la formation professionnelle, et dans la formation de la personne?

11 Si l'on reconnaît aujourd'hui que le plurilinguisme semble être la solution la plus raisonnable au sein de l'Union européenne et que tout Européen aura l'obligation de maîtriser, outre sa propre langue, une ou deux langues de l'Union'², ne peut-on s'interroger sur la cécité ou plutôt la surdité qui gagne les responsables politiques et les institutions face au plurilinguisme actif, vivant qui s'incarne dans la diversité des populations habitant sur le sol européen, si l'on prend cet exemple?

Quels relais sont construits pour passer de la performance individuelle réelle, car vécue au quotidien, par des centaines de milliers de personnes, à la reconnaissance et à la valorisation de ces plurilinguismes, en tant que constitutifs de la richesse et de la vitalité d'un pays? On sait que les droits linguistiques du citoyen, qui ne se limitent pas aux langues nationales et officielles, sont encore fort peu défendus en Europe et dans le monde, comme le rappelle $\mathrm{C}$. Truchot ${ }^{3}$.

13 L'école primaire, premier échelon de la scolarité obligatoire, n'a-t-elle pas un rôle particulier à jouer dans cette valorisation ? L'enseignement d'un idiome étranger n'estil pas le moyen le plus concret pour provoquer un questionnement des institutions et de l'opinion publique sur le statut des langues et les choix linguistiques?

Les premières années de la scolarité obligatoire entraînant souvent des relations plus étroites entre les familles, les enfants et les enseignants, on peut penser que l'introduction d'une autre langue à l'école va créer une onde de choc conduisant à une prise de conscience progressive mais réelle de la richesse, de la diversité des 
phénomènes langagiers et linguistiques et des facteurs affectifs, cognitifs, sociaux et culturels que mobilise ce nouvel apprentissage linguistique.

De par la position qu'elle occupe, l'école primaire, en s'ouvrant à un enseignement diversifié des langues, pourrait devenir un lieu privilégié d'explication des politiques linguistiques, un lieu de médiation entre les décisions supranationales et les volontés politiques affichées, celles prises par exemple dans le cadre européen, et l'évolution de la société civile sur ces questions.

16 Le grand public ignore bien souvent le contenu des décisions qui sont prises dans ces instances supranationales. On peut se demander pourquoi la décision du 4 juin 1985, prise par le Conseil et les ministres des pays de la Communauté européenne d'alors « de promouvoir toutes les mesures appropriées pour que le plus grand nombre possible d'élèves acquièrent, avant la fin de leur scolarité obligatoire, une connaissance pratique de deux langues en plus de leur langue maternelle, une des deux langues devant être une langue officielle de la communauté européenne ", aujourd'hui élargie à l'Union européenne, n'est pas largement médiatisée.

17 Ce texte ne peut-il pas constituer la charte de référence pour construire une politique plus ouverte et plus souple sur le choix des langues à l'école?

\section{À propos du choix des langues à l'école}

18 Ce choix n'est pas neutre et ne peut être seulement guidé par la demande des catégories sociales les plus actives ou les plus dominantes socialement ni par les seules priorités d'une économie de marché.

19 Si la diversification linguistique se développe dans la société civile - elle est déjà sensible dans les échanges économiques, artistiques, universitaires et commerciaux en Europe où l'on s'efforce de communiquer dans la langue du partenaire - ne pourrait-il pas en être de même à l'école?

On peut faire le raisonnement que la mobilité scolaire, professionnelle et individuelle fera plus pour la diversification face à la situation de quasi-monopole de l'anglais qui est, à long terme, une situation difficile à préserver.

\section{L'anglais n'est pas incontournable}

21 Il faut mener une campagne d'informations sur les langues à l'école et ne pas se réfugier frileusement derrière le conformisme linguistique ambiant.

22 En effet, si l'on se refuse à rentrer dans «cette fuite en avant» dont parle C. Truchot ${ }^{4}$, on doit construire une réflexion objective sur la nature et le rôle de l'anglais, et sur l'espace qui lui revient.

On constaterait alors que cet espace qui revient à l'anglais (langue d'échange dans les colloques et congrès internationaux, langue d'accès à l'information scientifique et technique, langue de publication, langue de laboratoire) n'est nullement couvert par le curriculum scolaire. On peut certainement l'acquérir de manière plus fonctionnelle et plus efficace plus tard, à d'autres moments du parcours de formation postscolaire et professionnel (dans le cadre de l'université et de la formation permanente, par exemple). 

sur l'opposition « unification linguistique » versus «pluralité et diversification des langues ${ }^{7}$, mais il est certain que ces questions cruciales dans les mutations à accompagner doivent sortir des cercles de spécialistes pour être largement expliquées et débattues démocratiquement et faire évoluer les représentations. objectives sur les langues, leur diversité, leurs ancrages culturels et sociaux. Les langues ne sont pas des objets-produits ordinaires et innocents, il faut donc que ces choix soient aussi inscrits dans les histoires des communautés et des individus que sont les familles et les enfants qui fréquentent l'école. Cette approche rend incontournable une analyse des contextes scolaires et extra-scolaires et des situations de plurilinguisme qui souvent les caractérisent. début de la scolarité, me paraît une bonne chose car il va renvoyer à un champ référentiel complexe qu'il est utile d'expliciter. En effet, introduire un idiome étranger dans l'école demande un repositionnement des concepts /notions tels que langue maternelle, langue seconde, langue de l'école, langue d'enseignement, et fait émerger les parties englouties de l'iceberg. "Qu'est-ce qui permet d'affecter à une langue le qualificatif $d$ "'étrangère" ? " s'interroge L. Dabène ${ }^{8}$. Elle prend appui sur la remarque de J. Widmer ${ }^{9}$ :

«Le statut normatif de la langue maternelle définit en creux celui de la langue étrangère, étrangère au pays et à nous-mêmes ", pour proposer une définition: «On peut donc appeler langue étrangère, la langue maternelle d'un groupe humain dont l'enseignement peut être dispensé par les institutions d'un autre groupe, dont elle n'est pas la langue propre ".

Le choix du titre donné à cet article est aussi « en creux » afin de ne pas éluder cette complexité. L'émergence d'une prise de conscience des statuts, des fonctions et des rôles parfois contradictoires, toujours subjectifs, des langues, est salutaire. Elle exhibe leur non-neutralité, mais aussi leur vitalité. L'école a une fonction de légitimation à assurer dans ce domaine. 


\section{Diversification et souplesse des cursus}

31 Faire preuve d'imagination sur ces questions, c'est aller au-delà des propositions consensuelles tendant à introduire une deuxième langue vivante obligatoire dans le second degré. Pourquoi ne pas construire une charte des langues prévoyant la pratique obligatoire mais diversifiée de trois langues au cours du cursus, l'enseignement de l'anglais étant exclu du cursus de l'école primaire?

Par ailleurs, le système scolaire ne pourrait-il pas valider des pratiques de langues qu'il n'enseigne pas et donc attester la réalité et la diversité des langues comprises, parlées, écrites sur un territoire? Valoriser la diversité existante, n'est-ce pas faciliter la diversification des choix de langues à l'école? Une réflexion pourrait être conduite, dans ce sens, en France et en Europe.

La mise en place institutionnelle ne peut être conjoncturelle mais doit être organisée en tenant compte des capacités de l'institution scolaire à la gérer, donc forcément programmée sur le moyen ou le long terme.

34 Réfléchir à des parcours plus diversifiés, construire des cursus de langues moins monolithiques s'intégrant à un projet de politique linguistique plus large où l'école aurait sa place parmi un dispositif plus général, est une hypothèse à concrétiser.

\section{Des disciplines pas comme les autres}

35 Il faut cesser de faire croire que les langues sont des disciplines scolaires comme les autres. Les langues sont tout à la fois des objets formels et symboliques, structurés et structurants, culturellement marqués et multifonctionnels. Les apprendre et les pratiquer exige des efforts et du temps, et leur fréquentation est aussi une transformation.

Il faut donc aussi informer sur les types de contraintes auxquels elles soumettent ceux qui veulent les apprendre. Ces apprentissages sont longs, lents, partiels, fragmentaires mais, pour toutes ces raisons, ils participent à une formation de la personne sur le long terme car ils touchent à ses structures profondes (sensori-motrice, affective, sensorielle, cognitive). Donc, il n'est pas nécessaire de les apprendre toutes à partir d'un même modèle curriculaire, au même rythme, et pour développer les mêmes capacités linguistiques. Faisons, là encore, preuve d'imagination et de pragmatisme. On peut construire des cursus de langues différents, répondant à des besoins différents, donc exigeant des enseignements différents. Les quatre compétences linguistiques doivent être résolument dissociées.

Les compétences de réception, de compréhension orale et écrite doivent faire partie d'un premier bloc, qui lui-même pourrait ne pas viser un développement symétrique, selon les cursus. Des programmes construits sur de tels objectifs existent déjà, pour le primaire (option choisie en Suisse alémanique) et pour les adultes (programme Galatea).

Les compétences de production orale étant les plus difficiles à acquérir en contexte scolaire, il faudrait certainement en faire un des objectifs de la pratique dès l'école primaire, voire même dès l'école maternelle. Cela suppose bien sûr, d'autres aménagements d'horaire, de nombre d'élèves, de pédagogie. Les conditions 
d'organisation scolaire de ces enseignements freinent souvent la mise en place d'une pédagogie active des langues.

Il est évident que l'un des premiers verrous à faire sauter, c'est celui de l'uniformité des cursus de langues, dans leur organisation tant temporelle que structurelle. On doit pouvoir proposer des enseignements ayant des objectifs et des durées différentes. Et s'interroger sur quels types de compétences privilégier au début du cursus.

Un des atouts majeurs de l'introduction de l'enseignement d'une langue étrangère dans le cursus de l'école primaire est de conduire immédiatement à reformuler les objectifs, les rythmes et la place qu'il doit tenir dans les apprentissages fondamentaux. C'est, en particulier, une des raisons pour lesquelles la dimension interdisciplinaire de ce nouvel enseignement doit être affirmée et construite au plan didactique sachant que l'école primaire, comme l'école maternelle, s'y prêtent institutionnellement mieux que la suite du cursus.

\section{Repères didactiques}

Les obstacles à franchir pour construire la didactique d'un " enseignement primaire " des langues ${ }^{10}$ sont encore importants, sachant qu'il faut prendre en compte la spécificité du contexte, de ses atouts, mais aussi de ses limites, la spécificité du public destinataire - enfant et élève -, mais aussi la double spécificité de l'enseignant, à la fois spécialiste de langue et pédagogue du primaire.

Ce sont d'ailleurs les mêmes difficultés qui sont relevées sous forme de réquisitoire, par ceux qui sont hostiles à cette innovation. Ils dénoncent : un allongement excessif du cursus pouvant engendrer lassitude et démotivation pour les langues; une telle anticipation ne pouvant que renforcer la suprématie d'une langue (l'anglais) sur les autres, donc nuire au principe de diversité de l'offre; l'absence de continuité curriculaire et institutionnelle, réelle pour l'instant, difficultés que la succession de décisions politiques parfois contradictoires concernant la mise en place et les objectifs ne pouvaient que conforter ${ }^{11}$. Pour toutes ces raisons, ces auteurs souhaitent que le principe d'un «éveil au langage» soit développé à l'école primaire, comme propédeutique à un enseignement futur des langues étrangères.

43 L'approche "éveil au langage », inspirée du mouvement Awareness of Language créé par E. Hawkins ${ }^{12}$ en 1980, en Angleterre, se centre sur la place du langage dans le développement de la conscience métalinguistique. Si la démarche est fondée au plan éducatif, elle est très difficile à mettre en place de par la diversité des objets à manipuler (nécessité de différents corpus langagiers et linguistiques). Par ailleurs, on peut se demander si la conscience métalinguistique ne se construirait pas plus efficacement à travers les pratiques diversifiées et interdisciplinaires croisant la langue des apprentissages fondamentaux et l'idiome inconnu. De plus, nous constatons que cette démarche ne s'est pas encore concrétisée dans une méthodologie claire permettant d'élaborer des outils d'enseignement et de formation adaptés à différents contextes.

\section{Développer le plaisir pour apprendre les langues}

Il est utile de rappeler que pour tout enfant fréquentant l'école primaire, il n'y a pas de besoin existentiel pour apprendre cette langue, ni interne ni externe. Par contre, il est 
important de rappeler aussi que rien ne s'oppose, tant au plan de la phylogénèse que de l'ontogénèse, au développement de sa capacité à apprendre d'autres langues - appelée bilingualité par les psycholinguistes J. Hamers et M. Blanc ${ }^{13}$.

Par ailleurs, les recherches dans le domaine des neuro-sciences et des sciences cognitives nous apportent aujourd'hui des informations capitales sur notre potentiel cérébral et sur les capacités d'apprendre des êtres humains en développement que sont les enfants ${ }^{14}$. Nous sommes "nés pour apprendre ${ }^{15}$ et nous avons un équipement cérébral qui nous dispose à nous construire à travers un certain nombre de contraintes d'ordre génétique et d'ordre culture ${ }^{16}$ dont la langue fait partie.

Ce que nous savons aujourd'hui sur les étapes du développement du langage, à travers les apports des recherches de Piaget ${ }^{17}$, Wallon, Vygotski ${ }^{18}$, Bruner ${ }^{19}$, nous conduit résolument à promouvoir les conceptions socio-constructiviste et interactionnelle de l'apprentissage et de l'enseignement ${ }^{20}$.

47 Communiquer pour apprendre, interagir pour comprendre, être confronté à des tâches à la fois rassurantes et difficiles à réaliser avec ses pairs (les autres enfants de la classe) et l'adulte-enseignant, tels pourraient être les premiers principes d'une didactique des langues à l'école primaire.

Il faut avoir le courage de proclamer haut et fort, ce qu'aucune « instruction officielle n'a encore fait, qu'à ce stade du cursus, il s'agit d'abord de satisfaire les besoins primaires et les motivations générales de ce jeune public scolaire plutôt que d'apprendre et de parler une langue étrangère $»^{21}$.

\section{D'abord communiquer}

Comme le rappelle R. Titone ${ }^{22}$ : «L'enfant, entre trois et dix ans, est poussé par un fort besoin de communiquer et en conséquence par un vif intérêt pour l'acquisition de toute forme de langage verbal et non verbal. » Les enfants de l'école primaire sont prêts pour cet effort, à condition que nous soyons en mesure de respecter et de valoriser leur désir d'entrer en contact avec leur interlocuteur, que nous soyons attentifs à les rendre fiers des échanges et de la conscience qu'ils développent d'être confrontés à une langue différente de la leur.

Cette confrontation, dans un environnement positif et chaleureux, est un révélateur pour les enfants de leurs capacités d'effort, d'écoute, servies par une disponibilité psychique et physiologique immédiate et spontanée. Leur capacité à s'émouvoir et à s'identifier à ce qui les touche est le moteur de leur motivation, de leur désir de réussir. Le plaisir provoqué par cette réussite va engendrer à son tour le désir de réussir à nouveau, avec l'aide de l'adulte et puis tout seuls ${ }^{23}$. Il faut reconnaître que ce plaisir de faire va bien au-delà du besoin de comprendre, d'où leur intérêt pour les activités ludiques portant sur les aspects phoniques et phonétiques de la parole en langue étrangère.

51 Cette capacité perceptive mobilisée de manière ludique est un formidable levier pour acquérir avec moins d'effort consenti le noyau dur d'une langue dont parle J.-A. Ronda $^{24}$, en particulier les phénomènes phonétiques et prosodiques. "Une des responsabilités cardinales de l'école n'est pas de proposer des apprentissages pertinents mais encore de les proposer au meilleur moment. » Et l'école primaire est encore le bon moment pour jouer avec la langue comme matériau phonique. 


\section{Le goût immodéré pour les histoires}

Cette capacité encore non inhibée pour jouer avec les formes phoniques de la langue étrangère n'est nullement incompatible avec la construction du sens et la centration sur le contenu des messages.

Les récits, les histoires lues, expliquées avec l'aide de tous les codes disponibles non verbaux (images, gestes) et verbaux, ceux des codes linguistiques des langues en présence répondent également au besoin de raconter, et de se raconter, l'enfant ayant comme tout être humain « la possibilité d'inventer et pour inventer un futur, il faut pouvoir à chaque instant réinventer son passé. ${ }^{25}$

4 Il y a en chaque enfant un romancier parce qu'il y a en lui cette capacité de l'espèce humaine à raconter sa vie, à la mettre en scène. La langue étrangère va imposer sa propre forme d'organisation à travers la structuration des énoncés et des récits désirés et aimés, partagés. Pour découvrir, un jour peut-être, beaucoup plus tard que «c'est à l'intérieur du système de la langue et de lui seul que nous disposons des libertés de construction et de déconstruction, de mémoire et de projection dans l'avenir si illimitées, si dynamiques, si propres au caractère manifestement unique de la pensée et de l'imagination humaine qu'en comparaison, la réalité extérieure, quelle qu'elle puisse être ou ne pas être, n'est rien d'autre qu'une sphère inaccessible, de laquelle nous sommes privés à jamais » écrit G. Steiner ${ }^{26}$.

Cette fonction narrative et imaginaire, mise en œuvre dans les différents types de récits est un formidable tremplin pour développer la compréhension orale et écrite en langue étrangère, en vue de l'élargir progressivement à d'autres types de textes utilisés à l'école. Pourquoi l'enfant se poserait-il la question d'apprendre le russe ou l'italien? Cet idiome étrange et étranger n'est qu'un médium au service de son goût des histoires.

56 Il faut aider l'enfant à cultiver cette capacité à "faire émerger du sens ${ }^{27}$ à partir des rythmes, des sons, des mots, des organisations discursives venus d'ailleurs. On comprend pourquoi, à ce niveau du cursus, les compréhensions orale et écrite doivent être solidement développées en interrelation et que les compétences de reproduction orale doivent être sollicitées avec beaucoup d'attention et de bienveillance.

\section{Du côté des enseignants}

57 Un recadrage s'impose. Plus que des spécialistes de l'enseignement des langues étrangères, ils doivent être d'abord des pédagogues confirmés capables de développer les fonctions communicative, imaginaire, ludique dans la langue étrangère. Il est à noter que les enseignants de langues vivantes du second degré qui réussissent à l'école primaire ont souvent ces qualités diversifiées de communicateur et de pédagogue.

C'est donc l'enseignant généraliste de l'école primaire ou de l'école maternelle qui sera le mieux placé pour faire ce travail. Il connaît les besoins de l'enfant, sa psychologie, il a appris que «le savoir ne se transmet pas, il se construit, en particulier à travers les interactions socio-cognitives qui se jouent entre celui qui enseigne et celui qui apprend $»^{28}$. Il est déjà meneur de jeux, conteur et narrateur, chanteur et musicien souvent, il est expérimentateur et créateur d'objets et d'activités pour servir les savoirfaire et les savoir-dire. Il a seulement l'impérative obligation de devoir le faire cette fois-ci à travers la gestion des usages des langues en présence. 
Une méthodologie de l'interaction verbale et de l'alternance codique est à construire. Il faut donc à ce niveau du cursus abandonner cette conception fonctionnaliste et instrumentaliste de l'enseignement de la langue et prendre appui sur une approche plus globale et multidimensionnelle, faisant appel à toute la sensorialité et permettant de satisfaire les besoins socio-cognitifs et affectifs des enfants.

La capacité à gérer de manière attentive, mais non démonstrative, les interactions nonverbales et verbales dans la communication n'est pas encore une compétence inscrite dans un programme de formation à la didactique des langues et pourtant elle ne relève pas de la seule intuition pédagogique; elle se développe à partir de techniques d'observation et des pratiques construites.

61 Cinq ans, voire même dix ans, c'est jeune pour une innovation de cette importance, c'est pourquoi il est souhaitable qu'elle s'enracine tranquillement au cœur de l'école et modifie sans violence les habitudes culturelles scolaires et extra-scolaires d'une région, d'un pays, d'une nation, d'un concert de nations.

On peut donc se féliciter que cette introduction des langues à l'école ne soit pas encore généralisée, voire banalisée. Cela veut dire, pour moi, que l'introduction des langues étrangères à l'école primaire n'est pas seulement une innovation éducative à construire, mais qu'elle est aussi un changement de regard sur l'apprentissage des langues et sur l'apprentissage tout court.

\section{NOTES}

1. Innovalangues, organisé dans le cadre du $14^{\mathrm{e}}$ Expolangues, 15-19 février 1996, Grande Halle de la Villetteà Paris.

2. Carton, J.-M. Delefosse, Les langues dans l'Europe de demain, Paris, Presses de la Sorbonne nouvelle, 1994.

3. «Les conditions du plurilinguisme en Europe ", in: Les langues dans l'Europe de demain, op. cit., p. 79-86.

4. Op. cit., note 2, p. 79-86.

5. J. Hamers, «Un modèle socio-psychologique du développement bilingue », Langage et société, 43, Paris, Maison des sciences de l'homme, 1998.

G. Da Silveira, J. Hamers, « Scolarisation et bilingualité en contexte africain : un défi ? ", Langage et société, 52, 1990.

6. Note d'information 95-96 du ministère de l'Éducation nationale, Les langues vivantes dans le second degré en 1994-1995.

7. D. Girard, « Pour une politique de diversification de l'apprentissage des langues étrangères », Les langues modernes, 77, p. 285-291.

M. Candelier, G. Hermann-Brennecke, Entre le choix et l'abandon : les langues étrangères à l'école, vues d'Allemagne et de France, Paris, Didier, Collection « Essais », Crédit, 1993.

8. Repères sociolinguistiques pour l'enseignement des langues, Paris, Hachette, Collection «f. références ", 1994.

9. J. Widmer «Identités linguistiques et contacts des cultures. Quelques remarques à propos des statuts symboliques ", Tranel 19, Neuchâtel, 1993. 
10. W. Maier, «Théorie et pratique de l'enseignement primaire des langues vivantes », in : « Quels modèles didactiques pour enseigner/apprendre une langue étrangère à des jeunes scolaires ", (dir. M. Garabédian), Études de linguistique appliquée, nº 89, janvier-mars 1993, p. 57-71.

11. L. Dabène, op. cit. note 8 et M. Candelier, G. Hermann-Brennecke, op. cit., note 7.

12. Awareness of Language : an Introduction, Revised edition, Cambridge University Press, 1987.

13. Bilingualité et bilinguisme, Bruxelles, Mardaga, 1984.

14. H. Trocmé-Fabre, J'apprends donc je suis, Paris, Éd. d'Organisation, 1987 et 1991

15. H. Trocmé, D. Garabédian, Né pour apprendre, vidéogramme en sept cassettes, Éd. IUT de la Rochelle/ENS de Fontenay-Saint-Cloud, 1995.

16. B. Cyrulnik, Les nourritures affectives, Paris, Éd. Odile Jacob, 1993 et Né pour découvrir, première vidéo-gramme de la série Né pour apprendre, op. cit., note 15.

17. J. Piaget, Le langage et la pensée chez l'enfant, Neuchâtel, Delachaux et Niestlé, 1923 et Où va l'éducation, Paris, Médiations, 1972.

18. L. Vygotski, Thought and Language, Cambridge MIT Press, 1962.

19. J. Bruner, Le développement de l'enfant, savoir faire, savoir dire, Paris, Presses universitaires de France, 1983 et Comment les enfants apprennent à parler, Paris, Éd. Retz, "Actualité pédagogique », 1987.

20. M.-L. Martinez, «Le socio-constructivisme et l'innovation en français ", Pratiques, $n^{\circ} 63$, septembre 1989, p. 37-62.

21. B. Mallet, «Imaginaire et langage : position du textuel dans l'apprentissage précoce d'une langue étrangère ", op. cit., note 10 .

22. "Dix thèses sur l'enseignement précoce des langues étrangères ", Europe plurilingue, Revue de l'association pour le rayonnement des langues européennes (ARLE), $\mathrm{n}^{\circ} 2$.

23. Op. cit., note 18 .

24. J.-A. Rondal, Annick Comblain, « Apprendre une langue étrangère avant neuf ans », Le français dans le monde, $\mathrm{n}^{\circ}$ 250, juillet 1992, p. 63-65.

25. J.-D. Vincent, Né pour innover, $6^{\mathrm{e}}$ vidéogramme de la collection Né pour apprendre, op. cit., note 15.

26. G. Steiner, Réelles présences : les arts du sens, Paris, Gallimard, « Essais », 1991.

27. F. Varela, Né pour créer du sens, $4^{\mathrm{e}}$ vidéogramme de la série Né pour apprendre, op. cit., note 15.

28. Op. cit. note 20 .

\section{RÉSUMÉS}

Apprendre une langue prend du temps et participe à la formation de la personne. Dès l'école primaire, il convient donc de répondre au besoin de communiquer, très important chez l'enfant, grâce à des enseignants généralistes capables de développer les fonctions communicative, imaginaire et ludique, dans une langue étrangère. 
INDEX

Index géographique : Europe

Mots-clés : école primaire, enseignement des langues, enseignement primaire, langues étrangères, enseignement précoce des langues vivantes

\section{AUTEUR}

\section{MICHÈLE GARABÉDIAN}

Maître de conférences au Centre de recherche et d'étude pour la diffusion du français (CRÉDIF), École normale supérieure Fontenay-Saint-Cloud, France. 